\title{
3D Reconstruction of Bacterial Cells Provides Basis for Modeling their Cellular Processes and Molecular Networks.
}

\author{
Alice Dohnalkova*, Mason Mackey**, Harold Trease*, Jim Fredrickson* and Mark Ellisman** \\ * Pacific Northwest National Laboratory, Environmental Microbiology, Richland, WA 99352 \\ ** National Center for Microscopy and Imaging Research, University of California, San Diego
}

One of the main challenges in the post-genomic era is to make use of the genome information for understanding the biological responses and signaling pathways in cells. For more than a decade, soil bacteria of Shewanella sp. recovered from diverse environmental sources have been intensively studied for their ability to reduce Fe(III), as well as a number of heavy metals and radionuclides, in attempt to find fundamental understanding of the process of electron transfer and metal reduction, which could ultimately lead to developing a technology for immobilization of these contaminants in the subsurface [1]. S. oneidensis strain MR-1, an organism with considerable metabolic versatility serving as a model Fe(III)-reducing microbe, got recently into spotlight of high throughput methods of molecular biology; its complete genome is known now, and progress is being made on identifying parts of molecular network responsible for electron transport and metal reduction. However, an array of proteome analyses has to be coupled with methods providing basis for computational simulations of gene regulatory networks and metabolic processes applied to a spatially reconstructed MR-1 cell. Several microscopy methods including multi-photon confocal microscopy coupled with AFM provide complementary information to build this model. Electron tomography has been a method of choice for obtaining the high resolution volume of images for creating a mesh-based representation of the virtual microbial cell with accurate quantitative geometrical features.

Bacterial cell of MR-1 with its typical dimensions of about 0.5 microns in diameter, and around 1.5 $\mu \mathrm{m}$ in length is on the verge of feasibility to perform a tomographic projections of an intact cell. However, the approach of electron tomography of thick sections of cultured cells processed for TEM was selected rather than the reconstruction from individual ultra thin serial sections. Since it was critical for our purpose to preserve the bacterial morphology and dimensions with the most possible accuracy, traditional aldehyde fixation notorious for cell contraction was replaced by high pressure freezing and subsequent freeze substitution methods. Blocks were sectioned to a thickness of 250 to $500 \mathrm{~nm}$, and imaged on NCMIR's JEOL 4000 operating at $400 \mathrm{kV}$. Images of sixty TEM tomographic projections of $S$. oneidensis MR-1 taken in the interval of -60 to +60 degrees were collected; volume was then reconstructed, and geometry extracted for creating a mesh-based model.

Thanks to NCMIR's TEM remote operation capability, the highly sophisticated instrument is available to outside users. Connection to the NCMIR Teleportal is based on Java platform, and its comprehensive microscope operation and image analysis links allow sharing resources from different research facilities.

Our future direction is to get an insight into MR-1 protein function by investigating the spatial distribution and quantitation of specific proteins by cryo immunogold techniques, and study the dynamics of more complex systems like microbial communities and biofilms.

[1] Lovley D.R. Dissimilatory metal reduction. Ann Rev Microbiol 47:263-290 (1993) 
[2] Part of this research was conducted in the EMSL, a national user scientific facility sponsored by DOE, located at PNNL in Richland. Many thanks to Galen Hand (NCMIR) for assistance with high pressure freezing of cell suspensions.

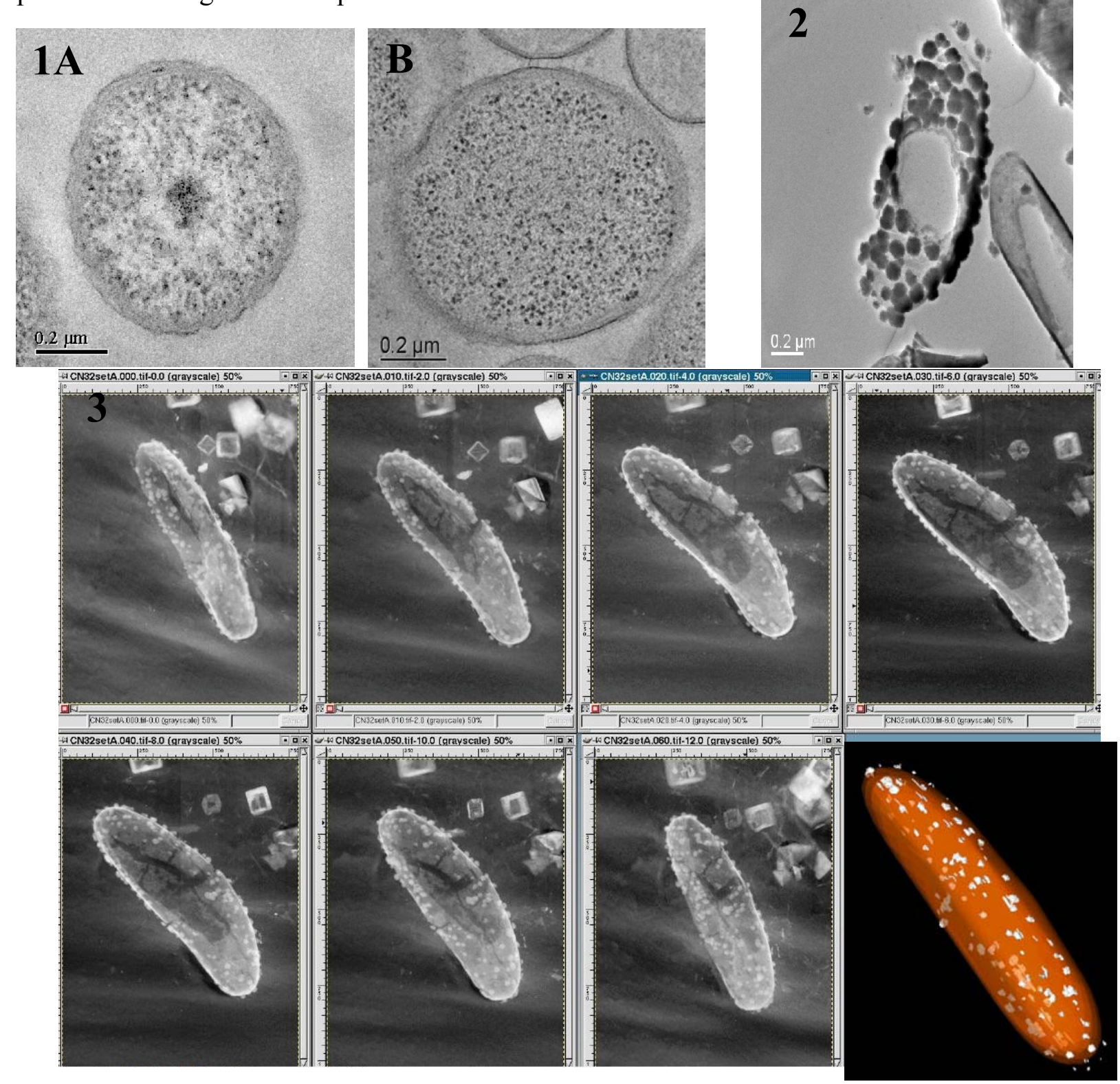

Fig 1. Cell morphology has been remarkably improved by high pressure freezing and freeze substitution processing (B) in contrast with conventional chemical fixation (A). Both A and B are untreated cells.

Fig.2. Thin section of $S$. putrifaciens $\mathrm{CN} 32$ forming a heavy deposit of biogenic uraninite on the outer membrane and in the periplasm as a result of bacterial reduction in U(VI) environment.

Fig.3. Seven selected tomographic projections from the series of 60 images taken at $(-60$ to +60$)$ degrees, and the reconstructed cell model. White spots on the bacterial membrane represent electron-dense biogenic uraninite. 
https://doi.org/10.1017/S1431927603445790 Published online by Cambridge University Press 